\title{
Estudio térmico y estructural del proceso de solidificación de fundiciones de hierro con grafito $\operatorname{laminar}^{(\bullet)}$
}

\author{
P. Larrañaga* y J. Sertucha*
}

\begin{abstract}
Resumen
La fabricación de piezas de fundición de hierro con grafito laminar (FGL) es un proceso industrial extensamente utilizado en la actualidad. Por ello, el estudio de los procesos de solidificación en estos materiales y de los factores que influyen en la transformación líquido-sólido supone un conocimiento de gran utilidad a la hora de avanzar en el desarrollo tecnológico de este tipo de fundición. En el presente trabajo se estudian tres aleaciones de diferente composición química (hipoeutéctica, eutéctica e hipereutéctica) con el fin de analizar, comparativamente, las características estructurales del material a lo largo de la transformación líquido-sólido. Las muestras obtenidas se han monitorizado con el fin de registrar las curvas de enfriamiento y se han sometido a procesos de temple en distintos instantes durante la solidificación. Las aleaciones presentan diferencias dependiendo de su composición y potencial de grafitización. Los resultados obtenidos se discuten de forma comparativa, estableciendo modelos de solidificación que justifican el comportamiento de este tipo de materiales.
\end{abstract}

Palabras clave Fundición laminar; Solidificación; Carbono equivalente; Curvas de enfriamiento.

\section{Thermal and structural studies about the solidification process of grey cast irons}

Abstract

\begin{abstract}
The grey iron casting manufacture is an industrial process extendly used today. Therefore, the study of the solidification features obtained from this iron and the factors that have influence on such transition becomes a powerful tool in order to support the technological development of this type of material. In the present work, three inoculated alloys with different chemical compositions (hypoeutectic, eutectic and hypereutectic) have been selected so as to comparatively analyse the structural characteristics of the irons during the liquid-solid transformation. The behaviour of the samples has been controlled recording the cooling curves and then they have been quenched in order to study the structural characteristics at different stages of the solidification. The selected alloys show different solidification features as a function of the chemical composition and the corresponding nucleation potential. The obtained results have been discussed in terms of a comparative analysis, establishing a solidification model that explains the industrial behaviour of the alloys.
\end{abstract}

Keywords

Grey iron; Solidification; Carbon equivalent; Cooling curves.

\section{INTRODUCCIÓN}

La morfología grafítica de las aleaciones Fe-C-Si a las que no se adicionan elementos esferoidizantes es, en general, laminar. A este tipo de fundición también se le conoce como fundición gris, debido al color grisáceo que muestra la superficie de fractura. El grafito laminar aparece distribuido de forma aleatoria en la matriz metálica, formando un esqueleto interconectado como consecuencia de un crecimiento cristalino favorecido a lo largo del eje cristalográfico "a". La adición de pequeñas cantidades de elementos fuertemente desoxidantes y desulfurantes, como el magnesio o el cerio, favorece el crecimiento a lo largo del eje "c", dando origen a la morfología esferoidal ${ }^{[1-4]}$ (fundición nodular o esferoidal). Aunque esta alteración estructural ha supuesto un avance tecnológico importan$\mathrm{te}^{[5-7]}$, la fundición con grafito laminar (FGL) tiene una historia más dilatada que la esferoidal y sigue siendo un material extensamente utilizado en la

(•) Trabajo recibido el día 17 de Noviembre de 2009 y aceptado en su forma final el día 12 de Febrero de 2010.

* Ingeniería y Procesos de Fundición. Centro de Investigación Metalúrgica AZTERLAN. Aliendalde Auzunea nº 6, E-48200 Durango (Bizkaia). E-mail: jsertucha@azterlan.es. 
industria de automoción, maquinaria agrícola, estampación, bombas de agua, mobiliario urbano, sector máquina-herramienta, etc.

La gran versatilidad de aplicaciones de las FGL se debe a que combinan de manera eficaz propiedades tales como una adecuada colabilidad, gran maquinabilidad, elevada capacidad de absorción de vibraciones, alta conductividad térmica, bajo coste de fabri-

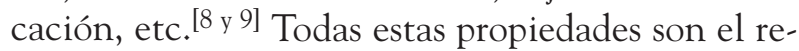
sultado de las características estructurales del material, las cuales son, a su vez, consecuencia del comportamiento de la aleación durante los procesos de solidificación ${ }^{[9 \text { y } 10]}$ y la posterior transformación en el estado sólido.

Dentro de la industria de automoción, los requerimientos establecidos en las propiedades mecánicas de la FGL han aumentado en exigencia con el fin de ampliar su funcionalidad y dar respuesta a la fabricación de motores diesel de elevada potencia, bajo consumo de carburante y mínimas emisiones de gases contaminantes. Habitualmente, la fabricación de estas aleaciones mejoradas supone la adición de determinados elementos químicos ( $\mathrm{Cr}, \mathrm{Mo}, \mathrm{Ni}, \mathrm{Cu}$, etc) Con ello se consigue modificar la estructura de la matriz y mejorar las propiedades mecánicas. Sin embargo, al igual que en el caso de la fundición esferoidal ${ }^{[11}$ y 12], la morfología de las láminas de grafito formadas en la transformación líquido-sólido también juega un papel esencial en las propiedades físicas del material. La capacidad de nucleación grafítica del metal fundido ${ }^{[13-15]} y$, por tanto, la forma, tamaño y distribución de las láminas de grafito precipitadas influyen directamente en el tipo de estructura obtenido a temperatura ambiente ${ }^{[8 \text { y } 16]}$. Por esta razón, el conocimiento sobre las etapas que conforman el proceso de solidificación y la evolución estructural de las fases en dicho período (especialmente el grafito) resultan de gran importancia a la hora de garantizar la correcta calidad de cada aleación ${ }^{[13]}$. En estas condiciones, la FGL puede seguir siendo competitiva en el mercado, proporcionando piezas de altas prestaciones obtenidas a partir de procesos de fabricación simples y en permanente desarrollo.
El presente trabajo forma parte de un estudio general sobre las transformaciones líquido-sólido en las fundiciones de hierro con grafito libre ${ }^{[11]}$ y pretende contribuir al desarrollo de la calidad de estos materiales. En esta ocasión, se estudian, comparativamente, las etapas del proceso de solidificación pertenecientes a tres aleaciones de FGL, de composiciones hipoeutéctica, eutéctica e hipereutéctica, utilizadas en la industria de automoción.

\section{EXPERIMENTAL}

La tabla I incluye la composición química y el carbono equivalente $\left(\mathrm{C}_{\mathrm{eq}}\right)$ de las diferentes aleaciones empleadas en este trabajo. Los contenidos de carbono y silicio obtenidos en cada caso permiten disponer de una aleación hipoeutéctica (FGL-HPO), una cercana al eutéctico (FGL-EUT) y una hipereutéctica (FGL-HPE). Esta selección permite analizar las diferentes posibilidades de solidificación existentes en el diagrama Fe-C-Si.

Todos los ensayos experimentales se llevaron a cabo en las instalaciones de TQC Technologies, S.L.U. En los tres tipos de aleación, el metal fundido se preparó introduciendo $15 \mathrm{~kg}$ de lingote, $15 \mathrm{~kg}$ de barras de acero y $20 \mathrm{~kg}$ de retornos (canales de llenado, mazarotas y copas de colada procedentes de la fabricación de piezas de fundición laminar) en un horno de inducción de media frecuencia $(250 \mathrm{~Hz})$ y $100 \mathrm{~kW}$ de potencia, con capacidad para $100 \mathrm{~kg}$ de metal fundido. Adicionalmente, en cada una de las cargas metálicas se adicionaron las correspondientes cantidades de $\mathrm{FeSi}$ (\% en peso, $\mathrm{Si}=74,2, \mathrm{Al}=1,7$ y Fe $=$ $24,1)$ y recarburante comercial de grafito. Tras la etapa de fusión de los materiales, se realizó una corrección de los contenidos de carbono y silicio con el fin de alcanzar las composiciones indicadas en la tabla I.

Las muestras de metal líquido se obtuvieron directamente a partir del horno de inducción, una vez finalizadas las labores de preparación de la aleación correspondiente. A continuación, dichas muestras se

Tabla I. Composiciones químicas de las aleaciones inoculadas (\% en peso)

Table I. Chemical compositions of the inoculated alloys used in this work (wt. \%)

\begin{tabular}{lccccccccc}
\hline \multicolumn{1}{c}{ Clave } & $\mathbf{C}$ & Si & $\mathbf{C}_{\text {eq }}{ }^{*}$ & Mn & $\mathbf{P}$ & $\mathbf{S}$ & $\mathbf{C r}$ & $\mathbf{C u}$ & $\mathbf{N i}$ \\
\hline FGL-HPO & 3,28 & 2,30 & 4,05 & 0,56 & 0,090 & 0,110 & 0,096 & 0,37 & 0,051 \\
FGL-EUT & 3,58 & 2,17 & 4,30 & 0,65 & 0,112 & 0,112 & 0,117 & 0,41 & 0,053 \\
FGL-HPE & 3,85 & 2,11 & 4,55 & 0,63 & 0,098 & 0,114 & 0,109 & 0,40 & 0,051 \\
\hline
\end{tabular}

${ }^{*} \mathrm{Ceq}=\% \mathrm{C}+\% \mathrm{Si} / 3$. 
colaron en el interior de tazas comerciales (Quik-cup ${ }^{\circledR}$ ) utilizadas para registrar las curvas de solidificación del material de acuerdo al procedimiento descrito anteriormente $^{[11 \text { y 17] }}$. Las muestras obtenidas tienen un módulo geométrico de 0,61-0,63 cm y su masa varía en el intervalo 340-360 g. Un termopar situado en la taza permitió registrar a lo largo del tiempo la evolución de la temperatura en la parte central de la muestra. La monitorización y tratamiento de las curvas de solidificación se realizó empleando el sistema Thermolan ${ }^{\circledR[17]}$. Esto permitió controlar las diferentes etapas de la transformación líquido-sólido en las muestras de metal. Fue posible realizar la caracterización estructural en cada una de estas etapas, introduciendo las correspondientes muestras en un recipiente con agua para efectuar un temple enérgico con el que se alcanzó la temperatura ambiente, en 4-5 s. Para cada aleación estudiada se templaron varias muestras con idéntica composición química y en distintos puntos del proceso de solidificación. Una de estas muestras permaneció sin que se aplicara enfriamiento alguno con el fin de registrar la curva de solidificación del material en su totalidad.

Antes de colar las tazas con el metal fundido procedente del horno de inducción, en el interior de éstas se introdujo 0,55 $\mathrm{g}(0,15 \%)$ de un inoculante industrial (\% en peso $\mathrm{Si}=75,4 ; \mathrm{Al}=1,4 ; \mathrm{Ca}=1,6$; Tierras raras $=0,7$ y $\mathrm{Fe}=20,9)$ con el fin de inocular todas las muestras empleadas en el presente estudio. Esta metodología reproduce el proceso industrial seguido habitualmente para la inoculación de piezas de fundición laminar.

Las muestras obtenidas tras efectuar los temples en agua, se cortaron con el fin de aislar la zona cercana a la ubicación del termopar. Posteriormente, estas zonas se prepararon para ser inspeccionadas metalográficamente. Se analizó la morfología, tamaño y distribución de las láminas de grafito junto con el tipo de constituyentes estructurales. La distribución de estas fases se determinó en forma de fracción de área (FA) medida en cinco campos metalográficos diferentes, con ayuda de un software específico basado en análisis de imágenes. Para llevar a cabo la caracterización microestructural, las superficies de inspección se atacaron con el reactivo Nital 5, durante un período de 5-10 s. Algunas de las muestras obtenidas se analizaron mediante la técnica EDX con el fin de determinar la composición química de las microinclusiones presentes en las zonas donde se ha formado el grafito.

\section{RESULTADOS Y DISCUSIÓN}

La figura 1 muestra comparativamente las curvas de solidificación para las tres aleaciones seleccionadas. En la tabla II se indican los parámetros de curva más significativos.

$\mathrm{Al}$ aumentar los contenidos de carbono y silicio para alcanzar la composición eutéctica, se obtiene una disminución en el valor del parámetro $\mathrm{T}_{\text {líq }}$ en la curva de solidificación, de acuerdo con lo esperado según el diagrama de fases Fe-C-Si. Por otro lado, al analizar conjuntamente las tres curvas se observa que

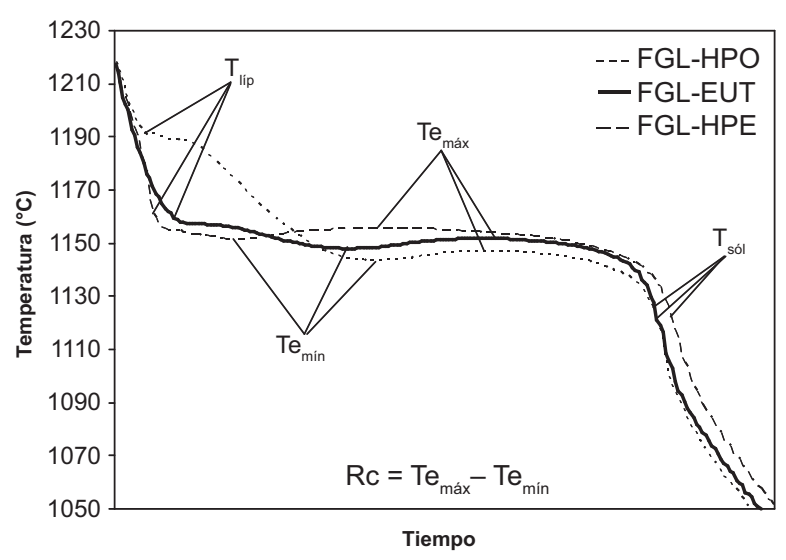

Figura 1. Curvas de solidificación pertenecientes a las tres aleaciones estudiadas.

Figure 1. Cooling curves registered from the studied alloys.

Tabla II. Parámetros de solidificación en las tres aleaciones seleccionadas

Table II. Solidification parameters for the selected alloys

\begin{tabular}{lccccc}
\hline \multicolumn{1}{c}{ Aleación } & $\mathbf{T}_{\text {líq }}\left({ }^{\circ} \mathbf{C}\right)$ & $\mathbf{T e}_{\text {mín }}\left({ }^{\circ} \mathbf{C}\right)$ & $\Delta \mathbf{T}^{*}\left({ }^{\circ} \mathbf{C}\right)$ & $\mathbf{R c}\left({ }^{\circ} \mathbf{C}\right)$ & $\mathbf{T}_{\text {sól }}\left({ }^{\circ} \mathbf{C}\right)$ \\
\hline FGL-HPO & 1190,7 & 1143,4 & 19,8 & 3,5 & 1124,9 \\
FGL-EUT & 1158,4 & 1148,0 & 14,7 & 3,7 & 1127,5 \\
FGL-HPE & 1160,2 & 1151,3 & 11,1 & 4,1 & 1133,7 \\
\hline
\end{tabular}

${ }^{*} \Delta \mathrm{T}=(1154+4 \times \% \mathrm{Si})-\mathrm{Te}_{\min }$. 
el incremento en el carbono equivalente también modifica el comportamiento del material durante el resto de la solidificación. La aleación hipereutéctica da lugar al valor más alto del parámetro $\mathrm{Te}_{\text {mín }}$, hecho que se relaciona con un mayor poder de nucleación grafítica ${ }^{[12 \text { y } 17]}$.

Las características estructurales obtenidas a lo largo de la transformación líquido-sólido son consecuencia de tres factores interrelacionados: la velocidad de enfriamiento del material, su composición química y el poder de nucleación grafítica durante el proceso de solidificación. Los ensayos realizados en el presente trabajo permiten analizar muestras que enfrían a velocidades similares $\left(250-290^{\circ} \mathrm{C} / \mathrm{s}\right.$ antes de alcanzar la $\mathrm{T}_{\text {liq }}$ ) por lo que se ha conseguido minimizar la influencia directa de este factor. De este modo, al estar todas las muestras inoculadas con la misma metodología, las variaciones en el comportamiento de las aleaciones estudiadas deben estar provocadas, fundamentalmente por su composición y, en consecuencia, por la capacidad del metal para nuclear y permitir el crecimiento de las láminas de grafíto. Las figuras 2, 3 y 4 muestran la evolución térmica y microestructural de cada una de las fundiciones analizadas, indicando los instantes donde se efectúan los temples en cada caso, las correspondientes fracciones sólidas (FS) y las metalografías más representativas.

Los efectos del temple en el material son los provocados por la aplicación de un enfriamiento crítico, es decir, el metal líquido solidifica según el diagrama Fe-C metaestable y origina zonas con abundantes carburos de hierro eutécticos (ledeburita). Los agregados cristalinos de austenita $(\mathrm{Fe}-\gamma)$ se transforman en martensita con austenita retenida en su interior y el grafito permanece con la morfología del instante en el que se realiza el temple. La figura 5 muestra un detalle de estas fases en la muestra 4 perteneciente a la aleación FGL-HPE.

Por otra parte, se detecta la formación de áreas donde aparece grafito de tipo D (grafito de subenfriamiento) rodeando a las zonas donde coexisten la fase Fe- $\gamma$ junto con finas láminas de grafito interconectadas entre sí. El espesor de estas áreas es de aproximadamente 10-30 $\mu \mathrm{m}$.

Durante la aplicación de un enfriamiento crítico, el tiempo disponible para la difusión de los átomos de carbono se reduce debido a la elevada velocidad de solidificación. En estas condiciones, aquellas zonas líquidas en las que el poder de nucleación grafítica permite solidificar según el eutéctico estable dan lugar a áreas donde coexisten láminas de grafito de distribución tipo D y austenita. La velocidad de crecimiento de estas áreas (V) puede ser estimada de acuerdo con la ecuación (1) ${ }^{[18]}$ donde, $\Delta \mathrm{T}$ es el subenfriamiento y $\mathrm{K}_{\mathrm{g}}$ es una constante cuyo valor depende del tipo de aleación. En este caso, se ha seleccionado el valor de $\mathrm{K}_{\mathrm{g}}=4,1 \mathrm{\mu m}^{-1 / 2} \cdot \mathrm{s}^{1 / 2}$ para aleaciones de tipo Fe-C-Si ${ }^{[18]}$.

$$
\Delta \mathrm{T}=\mathrm{K}_{\mathrm{g}} \cdot \sqrt{\mathrm{V}}
$$

Teniendo en cuenta que el subenfriamiento necesario para la nucleación del eutéctico metaestable es, aproximadamente, $40^{\circ} \mathrm{C}$, se obtiene una velocidad de crecimiento de $95 \mu \mathrm{m} / \mathrm{s}$. De acuerdo con la velocidad de temple mencionada anteriormente, el período de solidificación es de, aproximadamente, 0,3 s en este tratamiento y el espesor calculado para las áreas con grafito de distribución tipo D es $29 \mu \mathrm{m}$. Por ello, parece posible asignar la formación de estas áreas a la aplicación del enfriamiento crítico sobre las muestras.

Al analizar la evolución estructural de las fases durante el período de solidificación se observa que las tres aleaciones estudiadas siguen un patrón que depende de la composición química. En la aleación hipoeutéctica (FGL-HPO), la transformación líquido-sólido se inicia con la formación de cristales de $\mathrm{Fe}-\gamma$ en el seno del líquido y progresa con el crecimiento de esta fase para formar una compleja red de dendritas (Fig. 2). En esta aleación, la formación del grafito se inicia en los bordes de la austenita, cuando se ha superado el primer cuarto de la solidificación (Fig. 2, FS = 0,28). Este retraso en la formación del grafito se atribuye al hecho de que la aleación hipoeutéctica tiene la menor capacidad relativa para nuclear dicha fase. Los análisis metalográficos realizados indican que las primeras zonas con grafito están constituidas por un área central donde la austenita contiene grafito tipo $A\left(\gamma+G_{A}\right)$ y una capa envolvente, de espesor inferior a $30 \mu \mathrm{m}$, en la que coexiste la fase $\mathrm{Fe}-\gamma$ con el grafito originado durante el proceso de temple $\left(\gamma+\mathrm{G}_{\text {D-temple }}\right)$.

En las etapas iniciales de la solidificación de la aleación hipoeutéctica, aún no se dispone de una capacidad efectiva para nuclear grafito por lo que, tras la formación de la fase $\mathrm{Fe}-\gamma$, la reacción eutéctica se inicia a temperaturas comparativamente más bajas (Fig. 1). Este hecho da lugar a un subenfriamiento más elevado (Tabla II). El estudio, con ayuda del microanálisis por EDX, de las zonas en las que se inicia la formación de las láminas de grafito, es decir, aquellas en las que el temple origina grafito tipo $\mathrm{D}$, revela la existencia de microinclusiones de compuestos tipo (Mn,X)-S. Estos compuestos favorecen la nucleación heterogénea ${ }^{[13}$ y 14$]$ y promueven la formación de pequeñas láminas en estas áreas específicas. La figura 6 muestra la presencia de estas microinclusiones en el entramado formado por el grafito y la fase Fe- $\gamma$. 


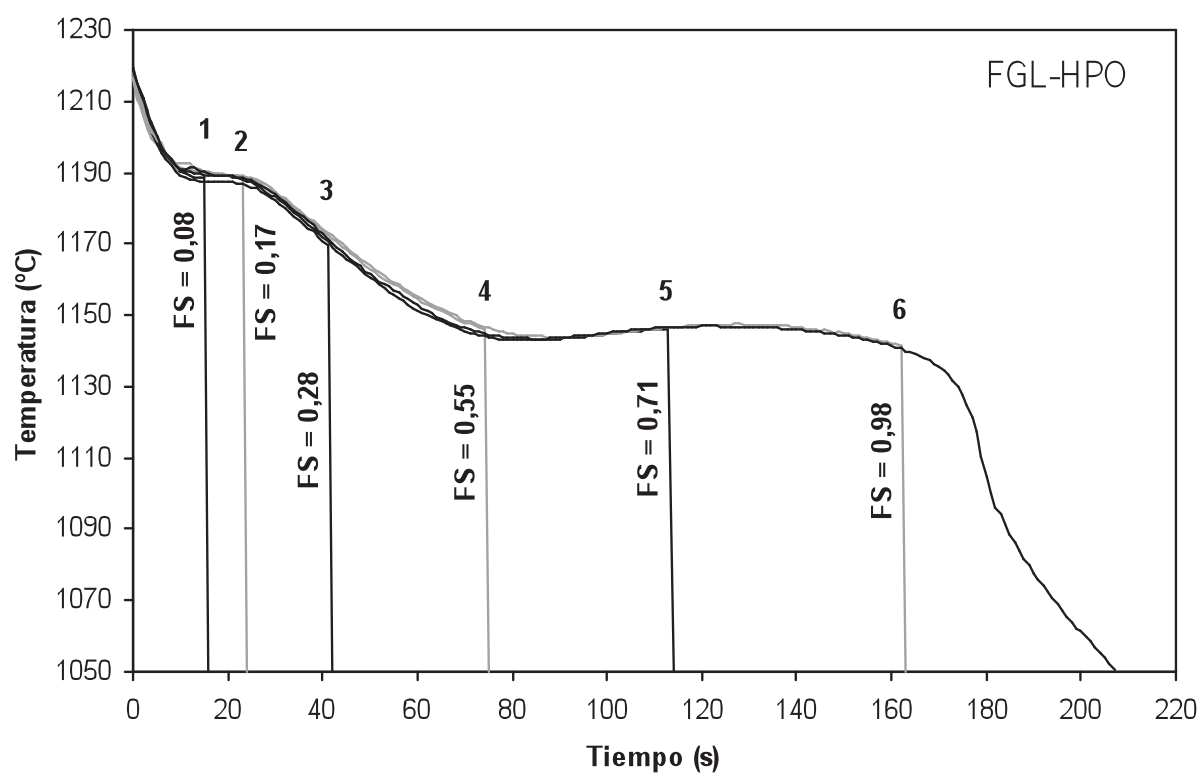

Muestra $224 \mathrm{~s}\left(1189^{\circ} \mathrm{C}\right)$

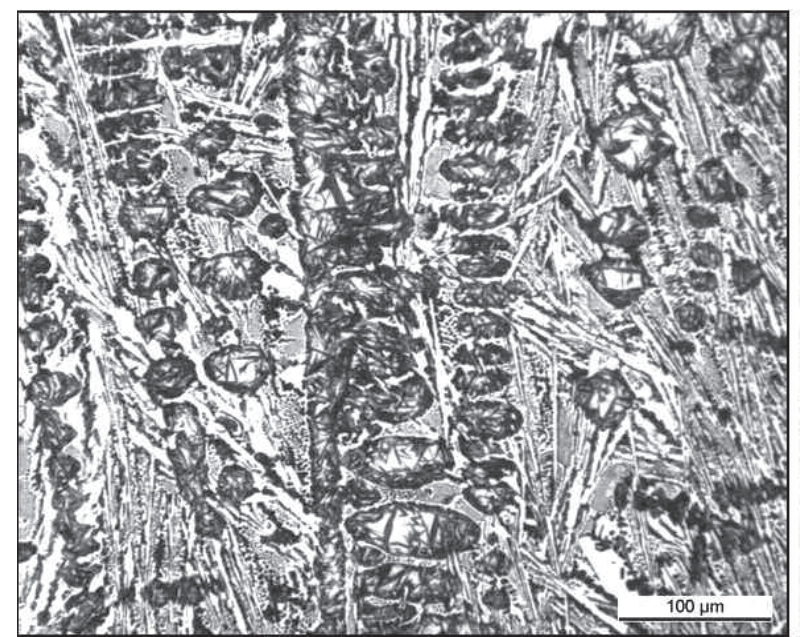

Muestra $5114 \mathrm{~s}\left(1146^{\circ} \mathrm{C}\right)$

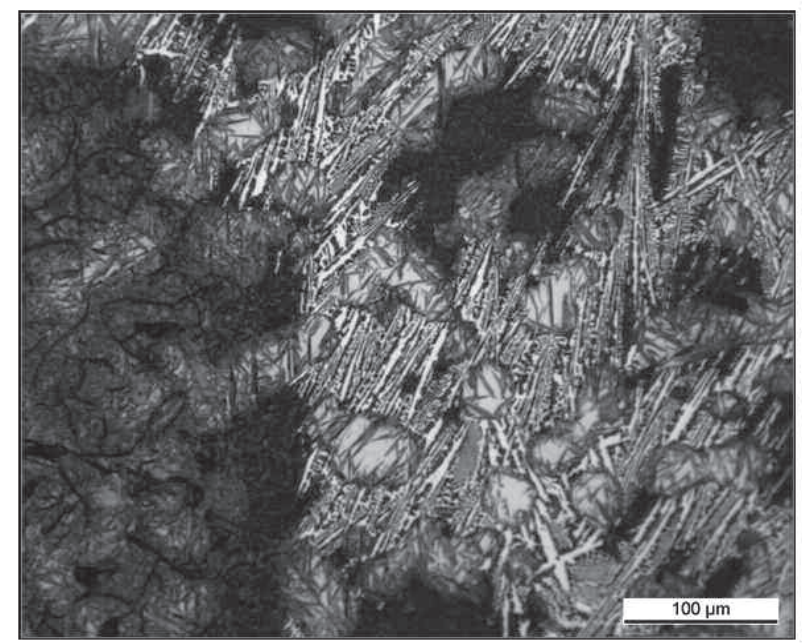

Muestra $3 \quad 42 \mathrm{~s}\left(1170^{\circ} \mathrm{C}\right)$

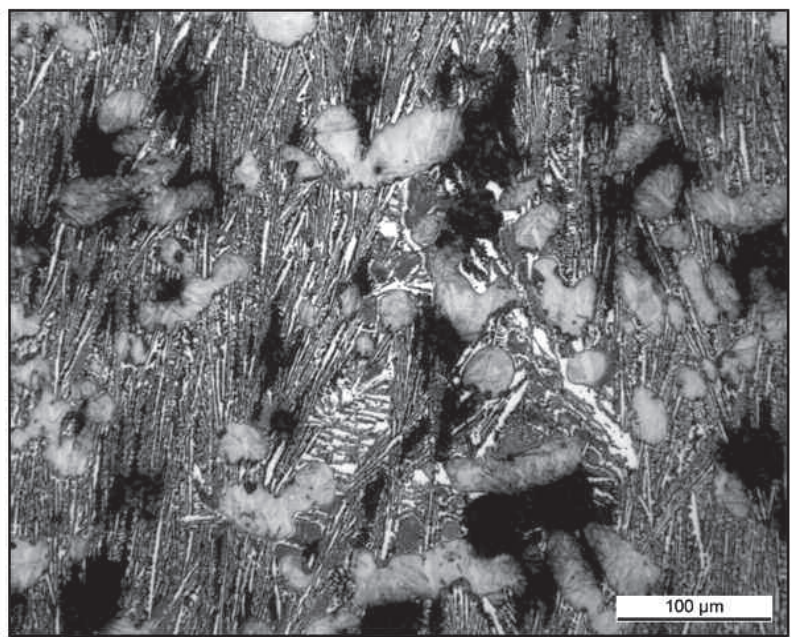

Muestra $6163 \mathrm{~s}\left(1142^{\circ} \mathrm{C}\right)$

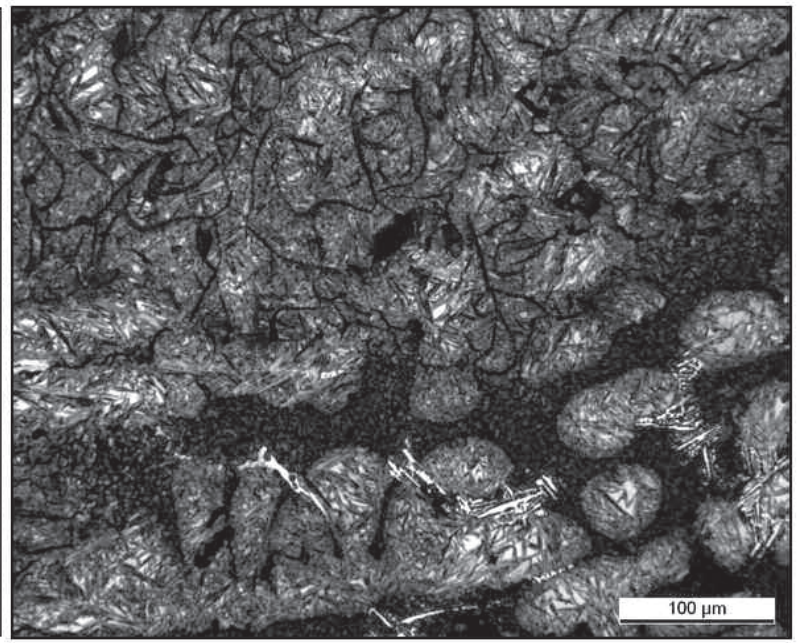

Figura 2. Evolución térmica y microestructural de las muestras obtenidas a partir de la aleación FGL-HPO.

Figure 2. Thermal and microstructural evolution of samples obtained from the FGL-HPO alloy. 
ESTUDIO TÉRMICO Y ESTRUCTURAL DEL PROCESO DE SOLIDIFICACIÓN DE FUNDICIONES DE HIERRO CON GRAFITO LAMINAR

THERMAL AND STRUCTURAL STUDIES ABOUT THE SOLIDIFICATION PROCESS OF GREY CAST IRONS

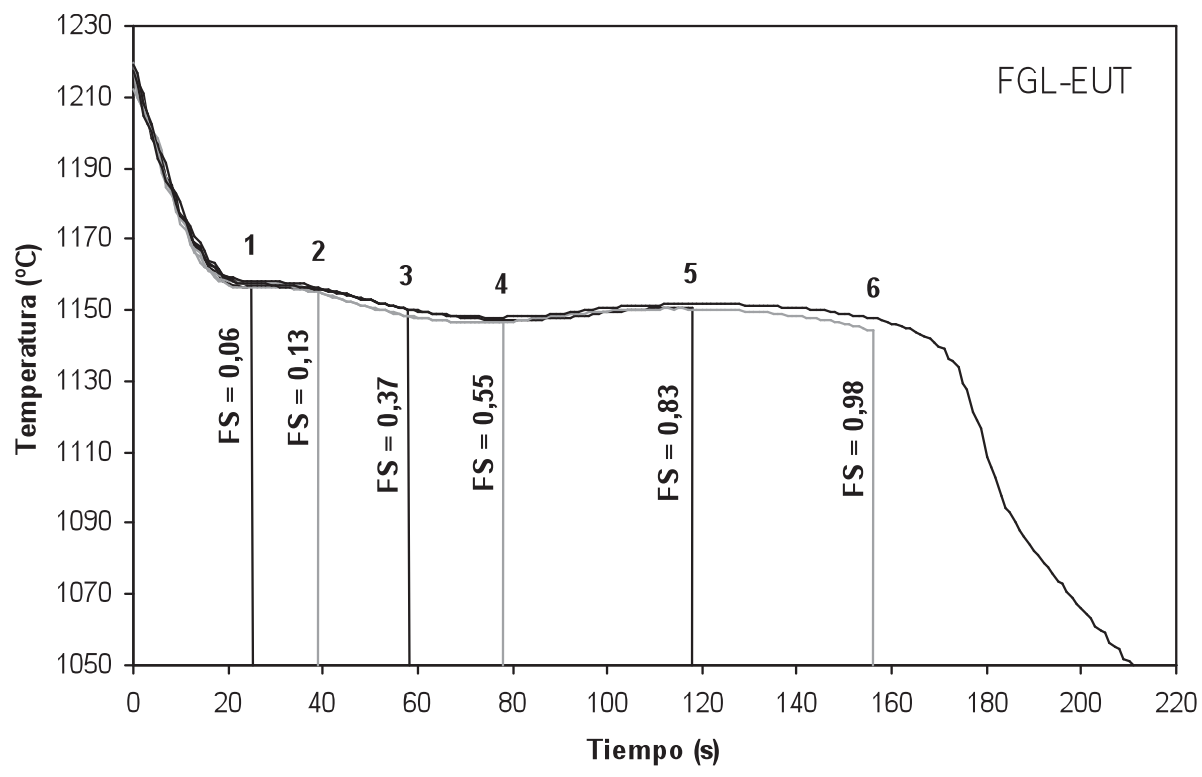

Muestra $126 \mathrm{~s}\left(1157^{\circ} \mathrm{C}\right)$

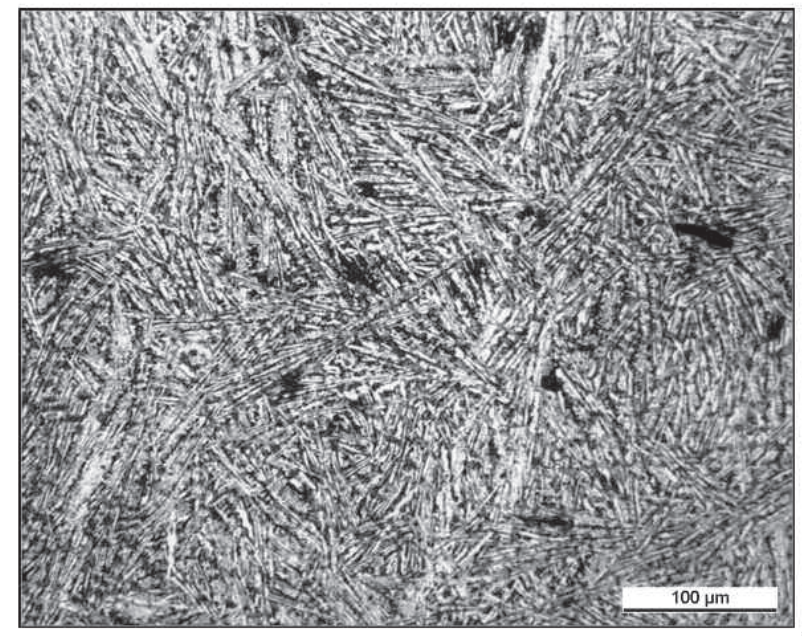

Muestra $5 \quad 119 \mathrm{~s}\left(1151^{\circ} \mathrm{C}\right)$

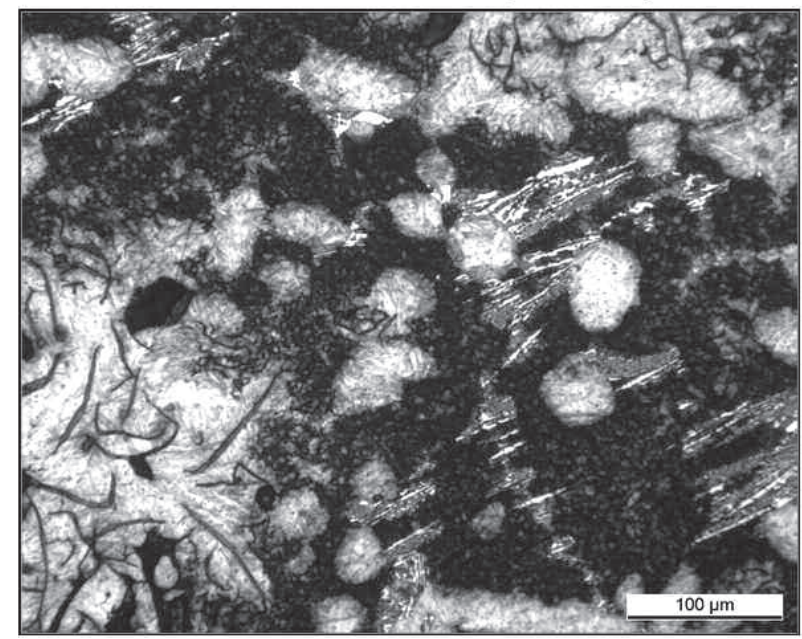

Muestra $359 \mathrm{~s}\left(1150^{\circ} \mathrm{C}\right)$

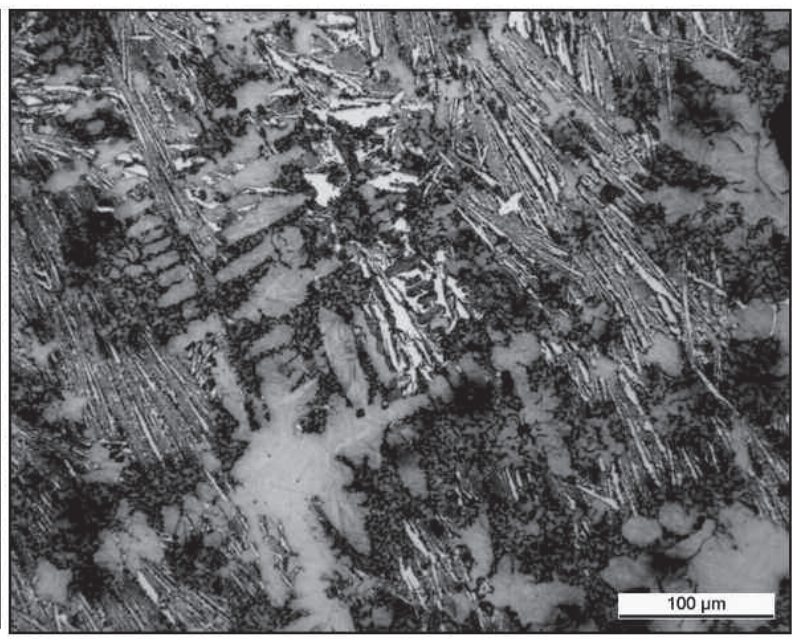

Muestra $6157 \mathrm{~s}\left(1145^{\circ} \mathrm{C}\right)$

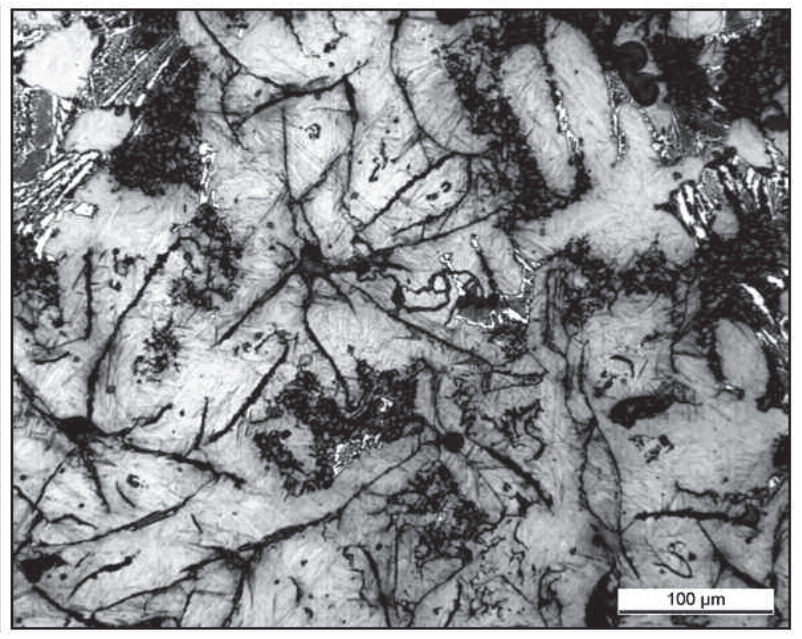

Figura 3. Evolución térmica y microestructural de las muestras obtenidas a partir de la aleación FGL-EUT.

Figure 3. Thermal and microstructural evolution of samples obtained from the FGL-EUT alloy. 


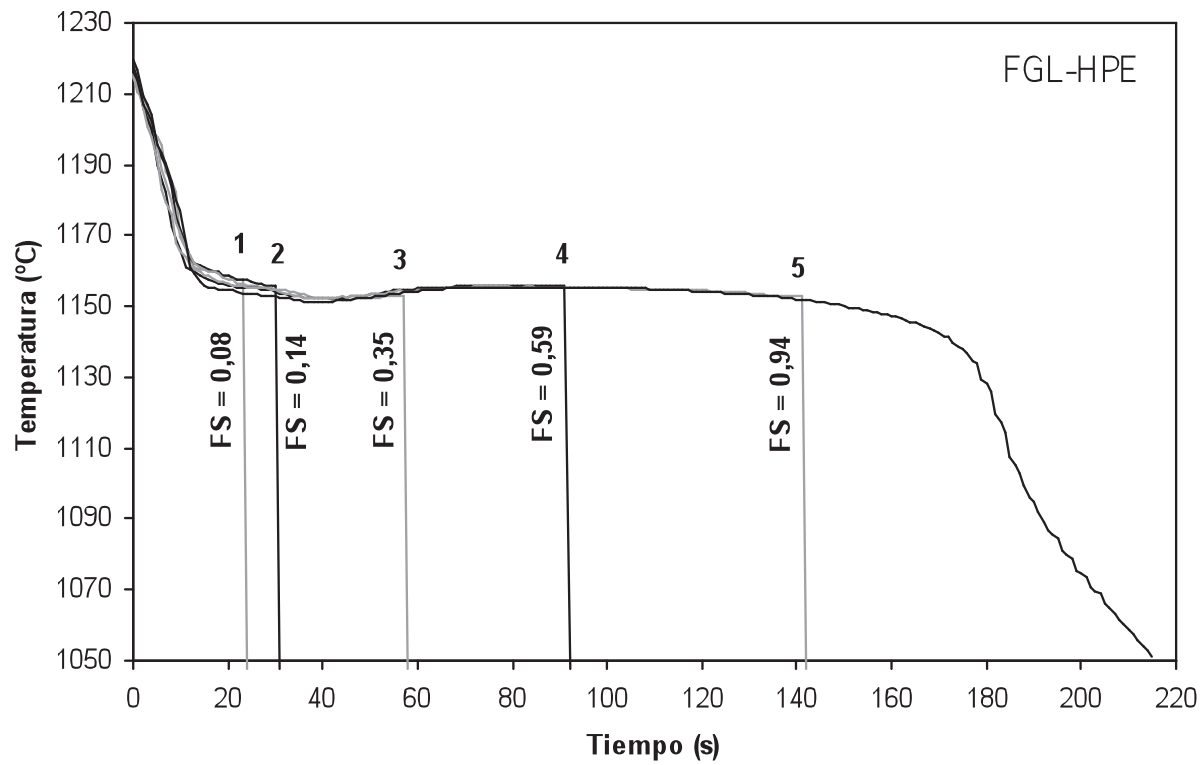

Muestra $124 \mathrm{~s}\left(1158^{\circ} \mathrm{C}\right)$

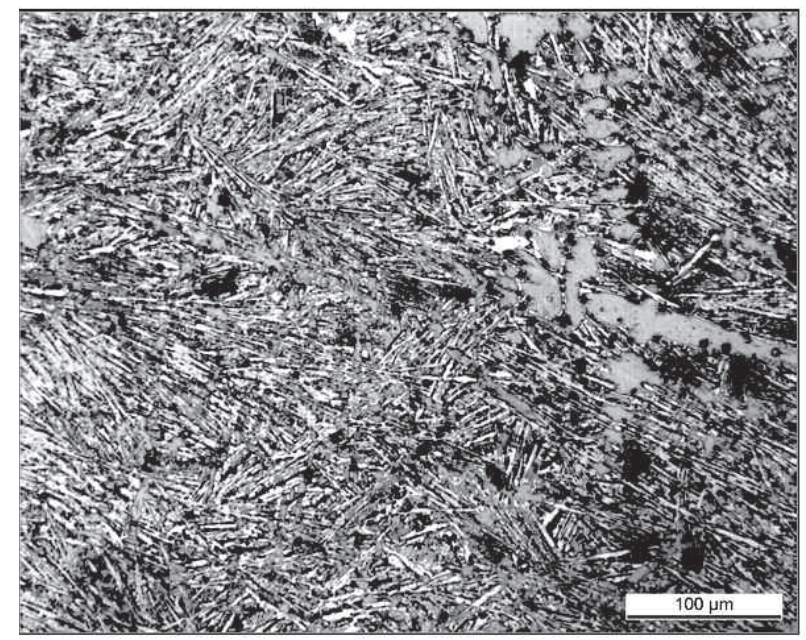

Muestra $492 \mathrm{~s}\left(1156^{\circ} \mathrm{C}\right)$

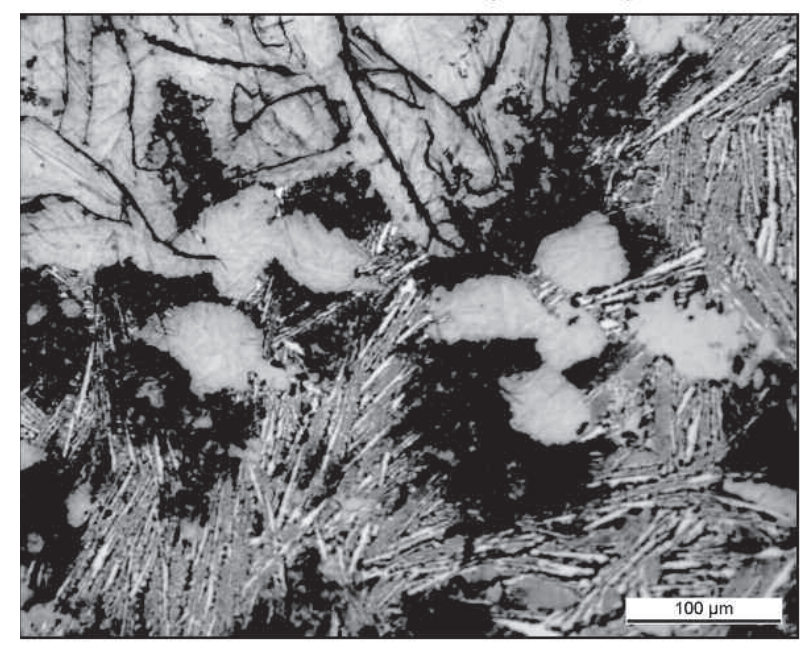

Muestra $358 \mathrm{~s}\left(1155^{\circ} \mathrm{C}\right)$

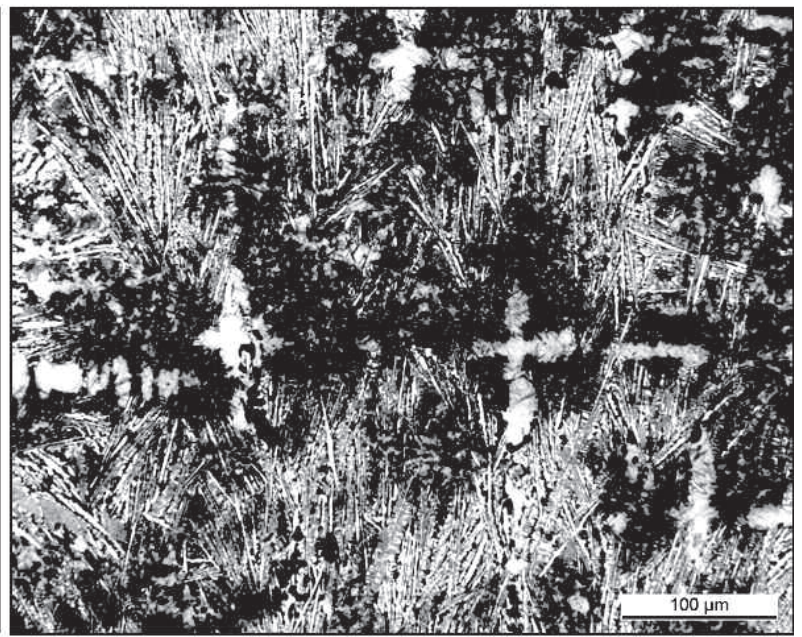

Muestra $5 \quad 142 \mathrm{~s}\left(1153^{\circ} \mathrm{C}\right)$

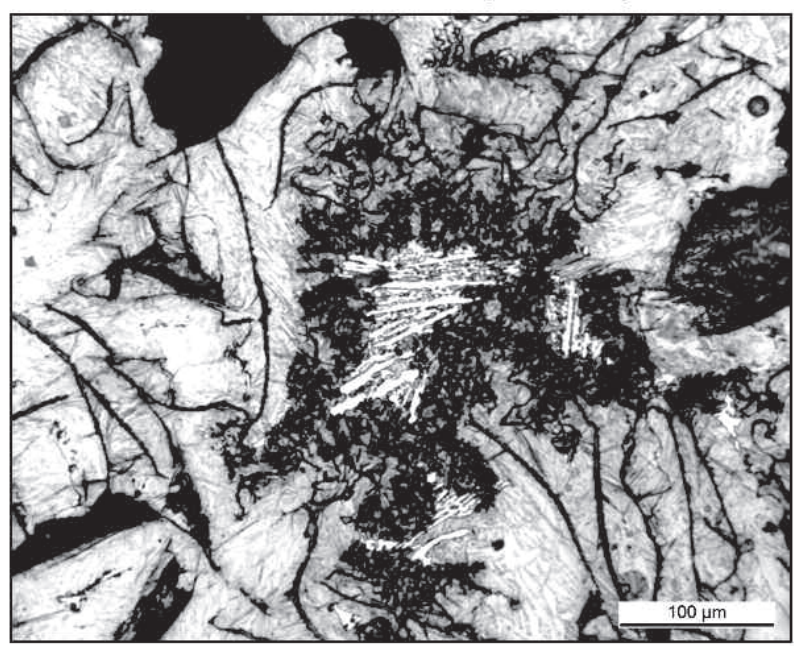

Figura 4. Evolución térmica y microestructural de las muestras obtenidas a partir de la aleación FGL-HPE.

Figure 4. Thermal and microstructural evolution of samples obtained from the FGL-HPE alloy. 


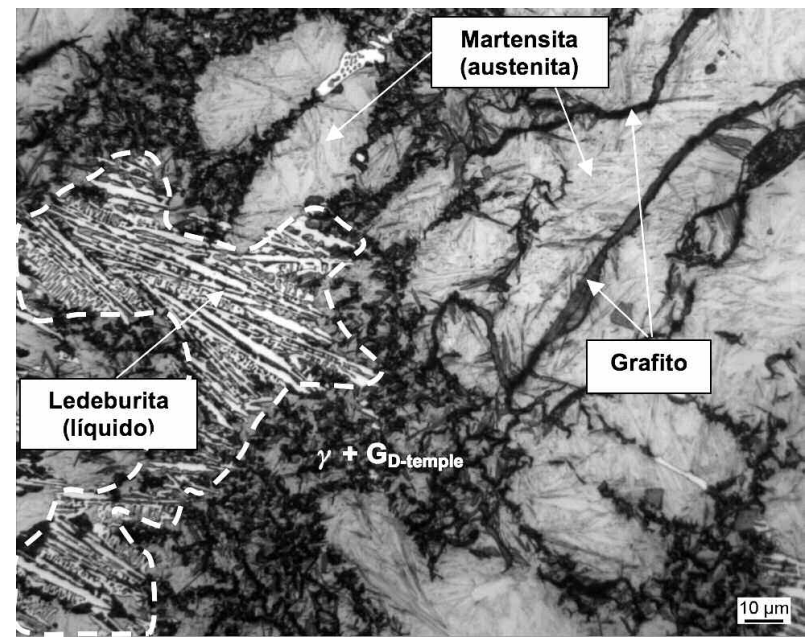

Figura 5. Fases estructurales obtenidas tras la aplicación del tratamiento de temple (muestra 4 en la aleación FGL-HPE).

Figure 5. Structural phases obtained after the application of the quenching treatments (sample No. 4 from the FGL-HPE alloy).

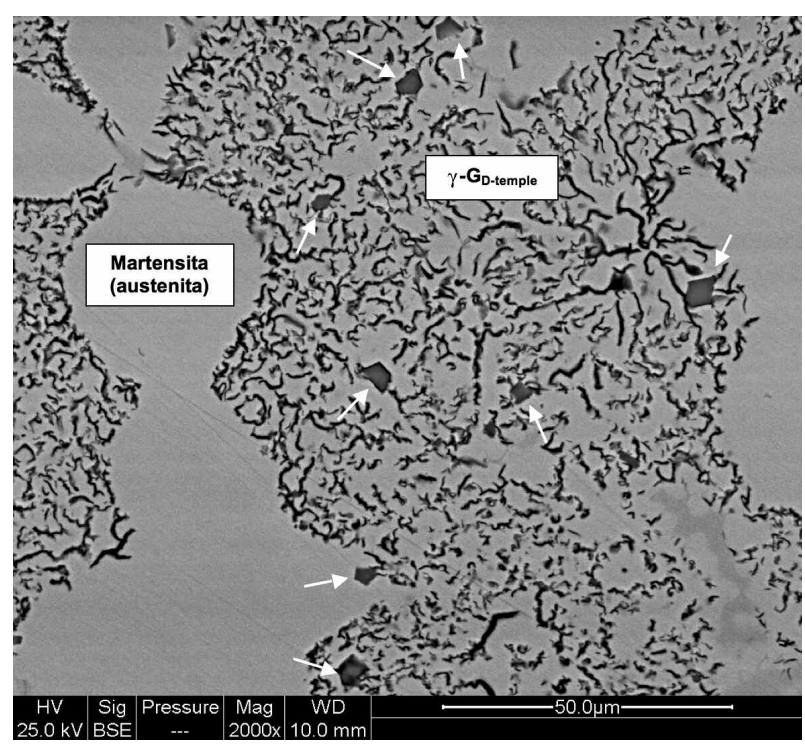

Figura 6. Las flechas señalan algunas de las microinclusiones de compuestos tipo (Mn,X)-S presentes en el interior de las áreas $\gamma-G_{D-t e m p l e}$ pertenecientes a la muestra 3 (FGL-HPO).

Figure 6. Arrows indicate some of the microinclusions of a $(\mathrm{Mn}, \mathrm{X})-\mathrm{S}$ compound included in the $\gamma$ - $G_{D \text {-temple }}$ area (sample No. 3 in FGL-HPO alloy).

Según avanza la solidificación (Fig. 2), disminuye la cantidad de líquido residual y los dominios constituidos por láminas de grafito tipo $\mathrm{A}$ incluidos en una matriz austenítica aumentan su tamaño. En el interior de éstos, las láminas de grafito continúan su crecimiento como consecuencia de la difusión del carbono a través de la austenita ${ }^{[10]}$. En la periferia de estas áreas $\gamma+\mathrm{G}_{\mathrm{A}}$ continúan observándose las zonas de cobertura $\gamma+\mathrm{G}_{\mathrm{D} \text {-temple }}$ generadas durante el enfriamiento crítico (Fig. 7). La transformación eutéctica finaliza cuando las áreas $\gamma+G_{A}$ ocupan todo el material.

En los procesos de fabricación de piezas reales, aquellas aleaciones con un poder de nucleación grafítica comparativamente bajo (similar al caso FGL$\mathrm{HPO}$ ) y/o las zonas de pieza sometidas a velocidades de enfriamiento más elevadas precisan mayores subenfriamientos para iniciar la formación del grafito. En consecuencia, se favorece la aparición de grafito tipo $\mathrm{D}$ en dichas zonas, tal y como se observa experimentalmente en las plantas industriales. Un ejemplo de ello es la fabricación de componentes de fundición laminar destinados a la fabricación de sistemas hidráulicos. En este tipo de piezas, las especificaciones requieren que el grafito sea mayoritariamente tipo D y E. Para ello, el metal líquido no debe superar la composición eutéctica y la colada se debe realizar en moldes metálicos (coquillas), para acelerar la solidificación del material.

Las aleaciones FGL-EUT (Fig. 3) y FGL-HPE (Fig. 4) solidifican con $\mathrm{Te}_{\text {mín }}$ más altas y menores subenfriamientos (Tabla II). El aumento en el carbono equivalente favorece la nucleación y el crecimiento del grafito y, en consecuencia, modifica el comportamiento del inicio de la solidificación. Aunque la formación de dendritas de Fe- $\gamma$ se ve desfavorecida en estas condiciones, éstas también nuclean y crecen. De este modo, la fase grafítica puede nuclear tanto en los bordes dendríticos como, directamente, a partir del metal líquido (muestras 1 y 3 en las figuras 3 y 4), iniciándose este proceso cuando la fracción sólida es inferior al caso de la aleación hipoeutéctica (FS = 0,05-0,12).

$\mathrm{Al}$ igual que en el caso hipoeutéctico, los dominios donde se forma la fase grafítica están constituidos por un área central con austenita y grafito tipo A (FGLEUT) o C (FGL-HPE) y una aureola ocupada por $\gamma+G_{\text {D-temple }}$. El espesor de esta capa exterior es mayor a medida que aumenta el contenido de carbono equivalente en la aleación. Este hecho se relaciona con una mayor saturación de carbono y silicio en el líquido que rodea la fase sólida ya existente en el momento de la aplicación del temple. La solidificación evoluciona incrementando las áreas $\gamma+\mathrm{G}_{\mathrm{A}}$ o $\gamma+\mathrm{G}_{\mathrm{C}}$ en detrimento del líquido residual y termina originando una matriz austenítica en la que el grafito se presenta con una distribución tipo A para la aleación FGL-EUT (muestra 6 en la figura 3) y tipo C para la FGL-HPE (muestra 5 en la figura 4).

La figura 8 representa, comparativamente, la evolución de las fases implicadas en el proceso de 


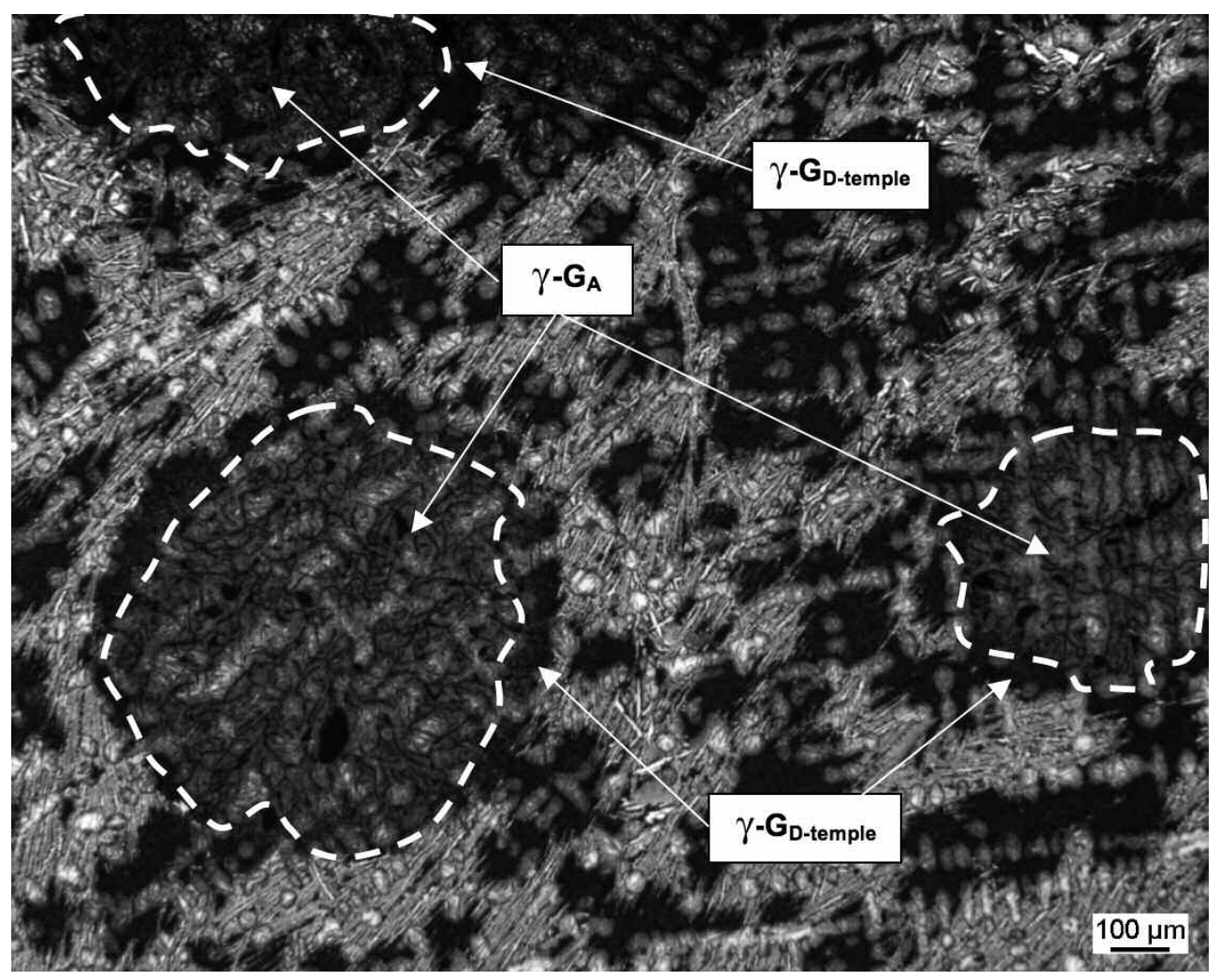

Figura 7. Detalle de las áreas $\gamma-G_{A}$ presentes en la muestra FGL-HPO.

Figure 7. Detail of the $\gamma-G_{A}$ areas formed in the FGL-HPO alloy.

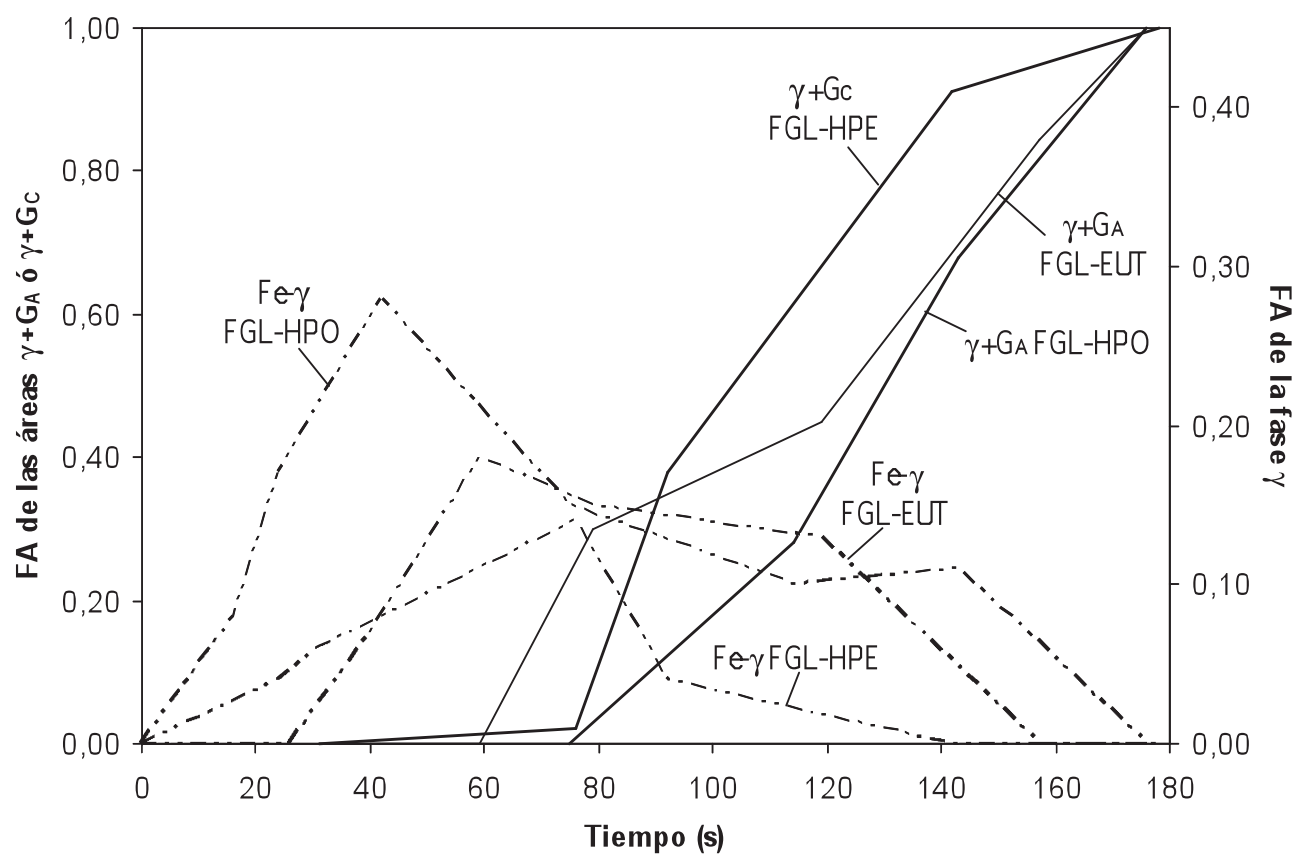

Figura 8. Evolución comparativa de las fases sólidas presentes en los procesos de solidificación.

Figure 8. Comparative evolution of the solid phases detected in the solidification processes. 
solidificación de las aleaciones seleccionadas. Se comprueba que un aumento en el carbono equivalente acelera la formación y el crecimiento de las áreas $\gamma+\mathrm{G}_{\mathrm{A}}$ (FGL-EUT) o $\gamma+\mathrm{G}_{\mathrm{C}}$ (FGL-HPE), es decir, las zonas donde nuclea y crece el grafito. Por otro lado, se retrasa la formación de la fase Fe- $\gamma$ y disminuye la cantidad de austenita proeutéctica no asociada a la fase grafito. Este comportamiento está directamente relacionado con la forma de las curvas de solidificación mostradas en la figura 1 .

Un aspecto descrito en la bibliografía ${ }^{[10}$ y 16$]$ y derivado del aumento en el carbono equivalente del metal es la formación y crecimiento de láminas de grafito en contacto directo con el líquido residual. En las tres aleaciones estudiadas en este trabajo, la formación de las áreas $\gamma+G_{\text {D-temple }}$ tiene lugar de forma que las láminas finas de grafito mantienen contacto con el metal que permanece líquido (Fig. 5). Sin embargo, el crecimiento de estas finas láminas ocurre a partir de los extremos de las láminas existentes en las áreas que ya son sólidas en el momento de la aplicación del temple $\left(\gamma+\mathrm{G}_{\mathrm{A}}\right.$ o $\left.\gamma+\mathrm{G}_{\mathrm{C}}\right)$. De este modo, puede pensarse que el crecimiento de estas láminas finas está relacionado con la deposición de átomos de carbono que se encuentran en el metal líquido y que son obligados a migrar como consecuencia de la imposición de un enfriamiento rápido. En condiciones habituales, la velocidad de enfriamiento del material debe permitir que las láminas de grafito tipo $\mathrm{A}$ o $\mathrm{C}$ crezcan alimentándose a partir del metal líquido que forma parte del frente de solidificación.

El conocimiento industrial indica que la capacidad de la fundición laminar para generar defectos de contracción aumenta al reducir el carbono equivalente de la aleación utilizada. Este hecho es consecuencia de los diferentes comportamientos mostrados durante la solidificación, de acuerdo a los resultados presentados en este trabajo. Al igual que en el caso de la fundición esferoidal ${ }^{[11]}$, una disminución en el carbono equivalente de la aleación reduce la cantidad de carbono disponible para formar grafito y el potencial de grafitización, favoreciendo la formación de austenita proeutéctica desde las primeras etapas de la transformación líquido sólido. Este hecho provoca la descompensación del balance contracción-expansión y aumenta el riesgo de formación de porosidades en las piezas. En el caso contrario, un aumento del potencial de grafitización en la aleación incrementa la expansión obtenida y se reduce la capacidad neta de contracción.

\section{CONCLUSIONES}

Los tres factores que influyen directamente sobre la transformación líquido-sólido en las fundiciones la- minares son: la velocidad de enfriamiento de la muestra, la composición química y el poder de nucleación. Estos dos últimos parámetros están íntimamente relacionados entre sí y modifican de forma importante las condiciones en las que se desarrolla la solidificación de las aleaciones utilizadas en este trabajo. El sistema Thermolan ${ }^{\circledR}$ ha permitido controlar cada una de las etapas que componen estos procesos de solidificación al efectuar temples selectivos en el material con el fin de analizar sus propiedades estructurales. Las principales conclusiones obtenidas son:

- En general, el proceso de solidificación de las FGL comprende las siguientes etapas:

- Formación de cristales de Fe- $\gamma$ en el seno del metal fundido una vez alcanzada la temperatura de inicio de la transformación líquido-sólido ( $\left.\mathrm{T}_{\text {líg }}\right)$.

- Crecimiento de las dendritas de Fe- $\gamma$ hasta el período eutéctico, donde se inicia la formación de las láminas de grafito a partir de los bordes de la austenita ya existente. En el caso de las aleaciones FGLEUT y FGL-HPE, las láminas de grafito pueden nuclear directamente a partir del líquido residual. En estos dos casos, también se forman y crecen dendritas de Fe- $\gamma$ según avanza la solidificación.

- Las láminas de grafito crecen insertadas en la austenita, alimentándose del carbono disuelto en el líquido sobresaturado que forma parte del frente de solidificación. Este metal líquido forma una zona que rodea las áreas sólidas y origina la formación del grafito de subenfriamiento durante la aplicación del temple.

- Crecimiento acoplado de la austenita y las láminas de grafito hasta completar la solidificación del material.

- En las aleaciones FGL-EUT y FGL-HPE, la fase grafito inicia su formación y crecimiento en etapas más tempranas que en la aleación hipoeutéctica. Este hecho supone una mayor compensación del balance expansióncontracción y una menor tendencia a la formación de rechupes y/o microrrechupes.

- La nucleación de las láminas de grafito está relacionada con la presencia de microinclusiones de compuestos tipo ( $\mathrm{Mn}, \mathrm{X})-\mathrm{S}$ en la matriz metálica.

- Bajo condiciones similares de enfriamiento, mayores carbonos equivalentes aumentan la capacidad de nucleación y crecimiento de las láminas de grafito a partir del líquido. Este hecho dificulta la formación de la austenita proeutéctica exenta de grafito. 
- La modificación del balance de formación entre las áreas que contienen las láminas de grafito y aquellas formadas exclusivamente por austenita define la capacidad de contracción neta del material durante su solidificación. Por otra parte, las alteraciones de este balance permiten que el material ya solidificado presente mayor riesgo de formación de grafito de subenfriamiento (especialmente en aleaciones sometidas a enfriamientos muy rápidos y/o con bajo poder de nucleación grafítica) o se origine grafito tipo C (aleaciones hipereutécticas).

\section{Agradecimientos}

Los autores de este trabajo agradecen la colaboración prestada por los responsables de TQC Technologies, S. L. U. a la hora de preparar las aleaciones estudiadas y todos los medios facilitados para la realización de los ensayos. Este trabajo se ha realizado en el marco del proyecto EKINBERRI 2007 (Diputación Foral de Bizkaia) con referencia EK-07-1.

\section{REFERENCIAS}

[1] C. R. Loper y K. Fang, AFS Trans. 116 (2008) 673-682.

[2] W. C. Johnson y H. B. Smartt, Metall. Trans. A 8 (1977) 553-565.

[3] A. Loizaga, J. Sertucha y R. Suárez, Rev. Metal. Madrid 44 (2008) 432-446.

[4] R. Suárez, J. M. Gutiérrez, A. Loizaga, P. Larrañaga y J. Sertucha, Rev. Metal. Madrid 45 (2009) 339-350.
[5] C. Labrecque y M. Gagné, AFS Trans. 72 (1998) 83-90.

[6] R. Ruxanda, L. Beltrán-Sánchez, J. Massone y D. M. Stefanescu, AFS Trans. 01-066 (2001) $1-12$.

[7] A. Varela, A. García, S. Zaragoza, J. L. Mier, F. Barbadillo y L. García, Rev. Metal. Madrid 44 (2008) 293-298.

[8] J. R. Brown, Foseco Ferrous Foundryman's Handbook, Ed. Butterworth-Heinemann, Oxford, Reino Unido, 2001, pp. 31-34.

[9] J. A. Pero-Sanz Elorz. Fundiciones Férreas: materiales para ingeniería. Ed. Dossat, Madrid, España, 1994.

[10] G. Rivera, R. Boeri y J. Sikora, Scripta Mat. 50 (2004) 331-335.

[11] P. Larrañaga, J. Sertucha y R. Suárez, Rev. Metal. Madrid 42 (2006) 244-255.

[12] J. Sertucha, R. Suárez, J. Izaga, L. A. Hurtado y J. Legazpi, Int. J. Cast. Met. Res. 19 (2006) 315322.

[13] A. Sommerfield y B. Tonn, Int. J. Metalcast. 3 (2009) 39-48.

[14] I. Riposan, M. Chisamera, S. Stann y T. Skaland, Proc. AFS Cast Iron Inoculation Conference, Schaumburg, Estados Unidos, 2005, pp. 31-41.

[15] I. Riposan, M. Chisamera, S. Stann, T. Skaland y M. O. Onsoien, AFS Trans. 109 (2001) 1.1511.162 .

[16] C. R. Loper y K. Fang, AFS Trans. 116 (2008) 665-672.

[17] P. Larrañaga, J. M. Gutiérrez, A. Loizaga, J. Sertucha y R. Suárez, AFS Trans. 116 (2008) 547-561.

[18] P. Magnin y W. Kurz, Metall. Trans. A 19 (1988) 1.965-1.971. 